\title{
Atenção psicossocial: ampliando o cuidado na construção de uma rede articulada em saúde mental
}

\author{
Psychosocial care: expanding care in the construction of an articulated network in mental health \\ Atención psicosocial: ampliar la atención en la construcción de una red articulada en salud mental
}

Recebido: 06/02/2022 | Revisado: 16/02/2022 | Aceito: 22/02/2022 | Publicado: 03/03/2022

\author{
Edilane Nunes Régis Bezerra \\ ORCID: https://orcid.org/0000-0002-8739-2638 \\ Universidade Federal da Paraíba, Brasil \\ E-mail: edilane_regis@hotmail.com
}

\begin{abstract}
Resumo
Os serviços substitutivos criados a partir da legislação regulamentadora da Reforma Psiquiátrica concorrem para a superação do modelo hospitalocêntrico, institucionalizado, partindo para um modelo de intervenção que privilegia a atenção integral e territorializada. O CAPS ocupa um lugar estratégico no processo da Reforma Psiquiátrica e na articulação da rede de atenção à saúde mental, aumentando a capacidade resolutiva de problemas de saúde mental pelas equipes locais. Este estudo pretende analisar as contribuições que o apoio matricial pode proporcionar para a ampliação de uma rede articulada em saúde mental. Realizou-se um estudo qualitativo, com a utilização da técnica de entrevistas semiestruturada, de acordo com alguns eixos temáticos. Enquanto método de análise, empregou-se a análise temática. Os resultados apontam que os CAPS precisam funcionar de forma articulada com a rede de serviços de saúde, assumindo um papel estratégico nessa articulação e no constante tecer da rede. Os serviços substitutivos não dão conta da Saúde Mental, por isso a Atenção Básica deveria entrar para ampliar a cobertura em saúde mental. O CAPS ocupa um lugar estratégico, de reordenar a saúde mental no território. Por fim, é essencial a articulação com a rede de saúde como um todo e com a comunidade, desempenhando uma assistência integral; Possibilitando a troca de saberes e de experiências, além do assessoramento às unidades básicas de saúde.
\end{abstract}

Palavras-chave: Atenção psicossocial; Saúde mental; Reforma psiquiátrica.

\begin{abstract}
The substitutive services created from the regulatory legislation of the Psychiatric Reform compete to overcome the hospital-centered, institutionalized model, starting with an intervention model that privileges integral and territorialized care. The CAPS occupies a strategic place in the Psychiatric Reform process and in the articulation of the mental health care network, increasing the capacity of the local teams to resolve mental health problems. This study aims to analyze the contributions that matrix support can provide for the expansion of an articulated network in mental health. A qualitative study was carried out using the technique of semi-structured interviews, according to some thematic axes. As a method of analysis, thematic analysis was used. The results indicate that CAPS need to work in articulation with the network of health services, assuming a strategic role in this articulation and in the constant weaving of the network. Substitute services do not cover Mental Health, so Primary Care should enter to expand mental health coverage. CAPS occupies a strategic place, to reorganize mental health in the territory. Finally, articulation with the health network as a whole and with the community is essential, providing comprehensive care; Enabling the exchange of knowledge and experiences, in addition to advising the basic health units.
\end{abstract}

Keywords: Psychosocial care; Mental health; Psychiatric reform.

\section{Resumen}

Los servicios sustitutivos creados a partir de la legislación reglamentaria de la Reforma Psiquiátrica compiten por superar el modelo hospitalario e institucionalizado, a partir de un modelo de intervención que privilegia la atención integral y territorializada. El CAPS ocupa un lugar estratégico en el proceso de Reforma Psiquiátrica y en la articulación de la red de atención a la salud mental, aumentando la capacidad de los equipos locales para resolver los problemas de salud mental. Este estudio tiene como objetivo analizar las contribuciones que el soporte matricial puede proporcionar para la expansión de una red articulada en salud mental. Se realizó un estudio cualitativo, utilizando la técnica de entrevistas semiestructuradas, según algunos ejes temáticos. Como método de análisis se utilizó el análisis temático. Los resultados indican que los CAPS necesitan trabajar en articulación con la red de servicios de salud, asumiendo un papel estratégico en esa articulación y en el constante tejido de la red. Los servicios sustitutivos no cubren la Salud Mental, por lo que debería entrar Atención Primaria para ampliar la cobertura de salud mental. El CAPS ocupa un lugar estratégico, para reordenar la salud mental en el territorio. Finalmente, es fundamental la 
articulación con la red de salud en su conjunto y con la comunidad, brindando una atención integral; Posibilitando el intercambio de conocimientos y experiencias, además de asesorar a las unidades básicas de salud.

Palabras clave: Atención psicosocial; Salud mental; Reforma psiquiátrica.

\section{Introdução}

No campo da saúde mental no Brasil, percebe-se, desde o surgimento do movimento da reforma psiquiátrica, em meados da década de 1970, uma pluralidade de saberes e produções. Dias e Amarante (2020) consideram que o paradigma da atenção psicossocial, referência prático-teórica construída a partir da reforma, é também potencialmente contra-hegemônico ao distanciar-se de uma perspectiva de saúde mental pautada exclusivamente na racionalidade biomédica, representada pela psiquiatria e pela hospitalização, que historicamente foram consolidadas como tecnologias de cuidado hegemônicas.

De acordo com Yasui (2010) o processo de reforma psiquiátrica no Brasil se caracterizou como uma transição paradigmática entre um modelo pautado na racionalidade médico-científica para um modelo de atenção psicossocial. O autor descreve uma ruptura radical com o modelo asilar até então predominante por meio do questionamento de seus fundamentos, conceitos e práticas. $\mathrm{Na}$ atenção psicossocial, a assistência não é mais entendida como um processo curativo, mas como produção de vida, de sentido e sociabilidade.

A atenção psicossocial busca ampliar as possibilidades de cuidado e assistência, ultrapassando inclusive o campo da saúde mental stricto sensu e dialogando com diferentes saberes e áreas de conhecimento. Assim, o fortalecimento da perspectiva da atenção psicossocial aponta para o entendimento de que o próprio processo de reforma psiquiátrica não se resume à psiquiatria, mas tem base em áreas diversas, como, por exemplo, a filosofia, as ciências sociais, as artes e o direito; e que portanto, precisa dialogar de forma ampliada com diversos saberes e conhecimentos (Dias et al., 2022).

As concepções e práticas de saúde, segundo o novo paradigma da Reforma Sanitária e Psiquiátrica, ao adotarem o território como estratégica, fortalecem a ideia de que os serviços de saúde devem integrar a rede social das comunidades em que se inserem, assumindo a responsabilidade pela atenção à saúde nesse espaço e incorporando, na sua prática, o saber das pessoas que o constituem. Assim, a atitude terapêutica deve basear-se na tutela, mas no contrato, no cuidado e no acolhimento (Rotelli, 2015).

Os estudos de alguns autores, Figueiredo e Onocko Campos (2008) e Dimenstein et al. (2009) apontam a importância da inserção da saúde mental na atenção básica, sendo necessária para o avanço da Reforma Psiquiátrica. Tal afirmação ressoa no fato de que $70 \%$ dos municípios brasileiros ter menos de 20 mil habitantes, não preenchendo critérios populacionais que justifiquem, em sua rede assistencial, dispositivos cruciais da política de saúde mental como os Centros de Atenção Psicossocial - CAPS. Desta maneira, o Ministério da Saúde vem construindo diretrizes e estratégias para que, em tais municípios, a rede de cuidados em saúde mental se estruture a partir da atenção básica, obedecendo ao modelo de redes de cuidado de base territorial e buscando o estabelecimento de vínculos e acolhimento (Brasil, 2005).

De acordo com estimativas do Ministério da Saúde e Organização Mundial da Saúde (2003), 3\% da população necessita de cuidados contínuos (transtornos mentais severos e persistentes) e mais de $9 \%$ precisam de atendimento eventual (transtornos menos graves). Ademais, os problemas de saúde mental ocupam cinco posições no ranking das dez principais causas de incapacidade no mundo, totalizando $12 \%$ da carga global de doenças, o que gera um custo substancial em termos de sofrimento, incapacidade e perda econômica.

As reflexões de Martín-Baró (1984) reforça a ideia de que a saúde mental é uma questão que não deve ser pensada de forma posterior à ideia de adoecimento mental. Neste aspecto, corrobora-se com os apontamentos de Lima (2018) quando sinaliza que não se trata de criar serviços e tratamentos que possam garantir um funcionamento satisfatório dos indivíduos, mas de criar e administrar uma cultura de humanização que possibilite o acesso de todos os indivíduos à esfera pública. 
Em 2005, a Secretaria Municipal de Saúde de Natal (SMS) estabeleceu alguns eixos para nortear a política de saúde mental municipal, sendo estas: a consolidação e expansão da rede extra-hospitalar, a articulação dos diversos dispositivos existentes, definindo como prioritária a articulação com a rede básica. Ademais, esta secretaria vem implementando o apoio matricial em sua rede de saúde. Neste cenário, o apoio matricial se incumbe de oferecer retaguarda assistencial e suporte técnico-pedagógico às equipes de referência, firmado no pressuposto de que nenhum especialista, de forma isolada, pode assegurar uma abordagem integral (SMS, 2005)

Para tanto, é necessária uma rede de serviços comunitários potente, que atue nos diversos contextos pelos quais circulem as pessoas em sofrimento psíquico. Dessa maneira, os CAPS se tornaram um dispositivo estratégico na operacionalização da atenção psicossocial, diminuindo significativamente as longas internações em hospitais psiquiátricos por meio de uma direção de cuidado ampliada, territorial e comunitária (Dias et al., 2022). O apoio matricial, além de ser um arranjo e uma metodologia de trabalho, também é considerado uma tecnologia em saúde como propõe Merhy (2006), tomando por eixo norteador o trabalho vivo em ato, força que opera em processo e em relações, o autor enfatiza três tipos de classificação das tecnologias envolvidas no trabalho em saúde, Tecnologia leve: tecnologias de relações do tipo produção de vínculo, autonomização, acolhimento e gestão como uma maneira de gerir processos de trabalho; Tecnologia leve-dura: saberes bem-estruturados que operam no processo de trabalho em saúde, como a clínica médica, a clínica psicanalítica, a epidemiologia, o taylorismo, o fayolismo; Tecnologia dura: equipamentos tecnológicos do tipo máquinas, normas e estruturas organizacionais (Merhy, 2006).

Por tudo isso, este estudo pretende analisar as contribuições que o apoio matricial pode proporcionar para a ampliação de uma rede articulada em saúde mental.

\section{Metodologia}

Trata-se de um estudo qualitativo, de cunho descritivo, Minayo (2010), sinaliza que a abordagem qualitativa, nas ciências humanas e sociais, preocupa-se com aspectos da realidade que não pode ser quantificado. Utilizou-se a análise de conteúdo e sua técnica temática como forma de tratamento das informações. Segundo Minayo (2010), a análise de conteúdo consiste em um conjunto de técnicas de análises de informações, utilizando-se de procedimentos sistemáticos e científicos de descrição do conteúdo das mensagens.

Para a análise de conteúdo, realizou-se uma pré-análise para a ordenação dos dados obtidos através das entrevistas individuais com os profissionais de saúde, bem como, através da leitura exaustiva dos textos referentes ao tema estudado. Para a análise das entrevistas elaborou-se um mapa temático, para o levantamento e sistematizações de temas que emergiram no transcorrer das falas dos participantes. Por fim, buscou-se estabelecer articulações entre os resultados obtidos e os referenciais teóricos da pesquisa com base no objetivo proposto.

Foi realizado o registro minucioso, reforçado com os comentários das equipes dos CAPS (simultaneamente ou após o término das entrevistas desenvolvidas). Também, se fez uso de um diário de campo, contendo notas descritivas e reflexivas além de relatos detalhados das situações.

As entrevistas foram realizadas com o auxílio de um gravador, com o devido consentimento dos participantes. As entrevistas individuais efetuadas (17 participantes), foram feitas à medida que cada indicação era aceita, nos diferentes serviços. As entrevistas tiveram duração média de 30 a 35 minutos. As transcrições das entrevistas foram feitas na íntegra. Os resultados foram obtidos através das análises minuciosas dos relatos das entrevistas.

As entrevistas foram realizadas com o auxílio de um gravador, com o devido consentimento dos participantes, foram feitas à medida que cada indicação era aceita, nos diferentes serviços. As entrevistas tiveram duração média de 30 a 35 
minutos. As transcrições das entrevistas foram feitas na íntegra. Os resultados foram obtidos através das análises minuciosas dos relatos das entrevistas.

O trabalho de campo foi realizado no município de Natal/RN, em quatro Centros de Atenção Psicossocial - CAPS na cidade de Natal/RN. Sendo: 02 CAPS II (Oeste e Leste) e 02 CAPSad (Leste e Norte). O CAPSi durante a realização do trabalho de campo, ainda não havia sido inaugurado. Vale ressaltar que os CAPS assumem um lugar estratégico na organização da rede de cuidados em saúde mental. Foram realizadas entrevistas individuais com os profissionais do CAPS II e CAPSad, totalizando 17 participantes, de diferentes categorias profissionais: médicos psiquiatras, psicólogos, assistentes sociais, enfermeiras, técnicas de enfermagem e nutricionistas.

\section{Resultados e Discussão}

Dialogando com as proposições de Leite e Paulon (2013), como a noção de saúde presente na nossa cultura ainda está bastante atrelada à ideia de adoecimento do corpo físico, a uma disfunção orgânica, este é, via de regra, o motivo que leva a população a procurar os serviços e os profissionais de saúde. Como, também, as formações para atuar em saúde ainda estão centradas no adoecimento dos órgãos e do corpo biológico, estas motivações encontram acolhida por parte dos profissionais de saúde que, comumente, restringem sua “escuta clínica” à queixa. Na perspectiva da Estratégia de Atenção Psicossocial, a produção de cuidado se dá pela via da identificação das necessidades singulares dos sujeitos, bem como dos recursos que estão disponíveis para atendê-las.

No presente estudo cerificou-se alguns obstáculos para se realizar a articulação da rede de serviços: o fato da qualificação dos recursos humanos, a escassez de referencial teórico, a ausência de uma rede de atenção básica eficaz, a inexistência de suporte dos serviços existentes, número insuficiente de profissionais. Ocasionando dessa forma, há uma demanda exagerada sobre as equipes, serviços superlotados, encaminhamento para outros serviços como forma de atenção, causando dificuldades de funcionamento das equipes.

Por se tratarem de problemas estruturais, estes devem ser percebidos no âmbito dos efeitos das políticas econômicas do governo brasileiro, que são reflexos da economia mundial, e só podem ser enfrentados mediante processos de envolvimento dos atores sociais na luta em defesa do SUS. Assim, verifica-se que a transformação da atenção depende da mudança de postura dos próprios profissionais, que muitas vezes não se veem como protagonistas. O processo mecânico e burocrático de gestão dificulta mudanças. Grande parte da equipe de gestores está apenas preocupado em adaptar métodos e modos de trabalho tradicionais. O que o SUS necessita hoje ultrapassa o limite adaptativo, entra no campo da reforma, da invenção e inclui um caminhar tenso pela micropolítica.

A organização dos serviços de saúde mental, com vistas a um olhar estratégico, vai ao encontro da nova forma de ver e assistir o indivíduo em sofrimento psíquico, pois pretende englobar os trabalhadores de saúde em geral, de saúde mental e, em específico, os usuários, familiares e, finalmente a comunidade que enseja a construção de uma prática de atenção à saúde mental mais justa, democrática e solidária. (Pitta, 2001).

Outro problema identificado é quanto ao serviço público, em que muitas vezes por questões políticas, se começa uma proposta e no decorrer do percurso não se dá continuidade. Isso acaba gerando incertezas e resistências nas equipes, porque acontece de a proposta ir caminhando a contento, vindo posteriormente a ser interrompida por alguma mudança no governo em que as pessoas envolvidas na proposta saem dos seus cargos e a pessoa que assume não dá continuidade à mesma.

Alguns dos entrevistados ressaltam a necessidade de que as mudanças ocorram em sistema de cogestão, ou melhor, acolhendo demandas de usuários, trabalhadores de saúde, especialistas, municípios, estados e, lhes oferecendo diretrizes, e submetendo as demandas a processos de discussão, construindo, desta maneira, um processo de mudança de modo mais 
interativo possível, em que todos possam colocar sua opinião sobre o processo de trabalho em saúde, sobretudo, como estão sendo realizadas as discussões acerca do AM em cada distrito sanitário.

Ao serem questionados sobre que tipo de demanda, da atenção básica, sugere a necessidade do trabalho do AM, uma das entrevistadas aponta que estão avançando nas discussões sobre a proposta da SMS acerca do AM, de modo que ainda não seja possível traçar muitas considerações a esse respeito. A entrevistada ressalta que o intuito é que os CAPS realmente possam chegar enquanto apoiadores para resolver as demandas de saúde mental nas unidades básicas. Porém, na sua opinião, estes sequer resolvem a demanda que chega até o serviço, de modo que ela questiona a efetividade de tal ação. Ela afirma a necessidade de se ter mais profissionais no serviço (CAPS) e, que, de fato, queiram se envolver com o AM, porque o AM para ser resolutivo em suas ações, demanda a participação de todos, de estar junto e fazer o AM acontecer e ampliar este trabalho na atenção básica. Observou-se na fala abaixo:

"Acho que é fundamental você poder contar, tá amparado e não no desamparo de rede, acho que é isso o risco que corre, os CAPS não trabalhar em território, não implementar esse princípio que é a territorialidade” (Assistente Social).

Outra questão colocada por outra entrevistada é a respeito do usuário que está em processo de alta e necessita de uma retaguarda, neste sentido, a rede básica poderia ser um mediador neste processo. Ou seja, o usuário tem que contar com a unidade básica para ajudá-lo a fazer o caminho, ajudá-lo a gerenciar as dificuldades, os problemas que venham a surgir após a alta. Como pensar a questão da inclusão social conjuntamente com a rede básica. Isso constatou-se na fala abaixo:

"Essa demanda é uma demanda necessária para a gente poder contribuir de forma efetiva, para que diminua este índice de crise para que realmente a gente contribua no processo de criar mecanismos de inclusão social”. (Assistente Social).

Percebe-se, então, que a partir do olhar dos profissionais dos CAPS, existem dois eixos básicos de ação/intervenção, na rede básica, que demandariam a atuação do AM: a necessidade de um apoio especializado por parte desses serviços e a retaguarda na reinserção social do usuário que venha a receber alta do serviço substitutivo. Este usuário seria encaminhado para a rede básica que, com o suporte do AM, faria essa retaguarda (Bezerra et al., 2008).

Constatou-se, em alguns relatos, a necessidade de capacitação permanente dos profissionais de saúde, para o cumprimento da política de saúde mental, pois só assim será possível concretizar transformações expressivas para a consolidação de ações e políticas voltadas a saúde mental, sem o engajamento dos atores sociais envolvidos neste processo não se pode alcançar resultados satisfatórios.

Diante disso, é importante ressaltar que o apoio matricial tem o potencial de atender algumas demandas apontadas pelos técnicos nos diversos níveis, como a falta de capacitação para as demandas de saúde mental, mas precisa ser feito de modo a não reforçar a lógica de saberes especializados, como pode ser constatado no trecho abaixo:

\section{A atenção básica quer da saúde mental um conhecimento mais amplo para saber lidar com o portador de transtorno mental" (Enfermeira).}

O Sistema Único de Saúde - e a Saúde da Família - tem prosseguido com a tentativa de disciplinar a demanda, ao afirmar a atenção básica como porta de entrada do sistema e não se preocupar em criar outros mecanismos de acolhimento e inclusão em outras portas de entrada efetivamente operantes (como são os prontos-socorros, por exemplo). A ideia da hierarquização e a definição de uma porta de entrada que deveria ser capaz de "resolver $80 \%$ dos problemas de saúde" correspondem a um esforço de racionalização, que esbarra exatamente na baixa capacidade das equipes de efetivamente 
resolverem problemas. Baixa resolubilidade que pode ser explicada de várias maneiras, entre as quais: a desarticulação entre a atenção básica e os demais serviços de saúde, já que os sistemas de saúde são organizados por níveis - atenção básica e atenção especializada (dividida em média e alta complexidade) - e não há mecanismos de articulação entre eles, que são operados e financiados segundo lógicas distintas. Bem como, o próprio perfil de formação dos profissionais, particularmente os médicos, que enfrentam profundas dificuldades para dar conta da complexidade dos problemas de saúde mais frequentes, tanto em seus aspectos estritamente clínicos, quanto em sua relação com as questões sociais: como as condições de vida e a violência, por exemplo. (Feuerwerker, 2005).

Associada a dificuldade em lidar com o usuário, os profissionais alegam que as equipes das UBS apontam a angústia por não poder dar uma resposta mais efetiva frente à demanda em saúde mental, uma vez que não se consideram despreparadas e julgam como necessário a realização de um apoio específico na área. Podemos verificar tal aspecto no relato da entrevistada, a seguir: A atenção básica demanda muitas vezes da saúde mental, uma ansiedade, uma angústia. Os profissionais se sentem imobilizados e não sabem como atuar frente a demanda de saúde mental" (Assistente Social).

Segundo Nascimento (2007), o apoio matricial é potencializador da desinstitucionalização e contribui para evitar internações arbitrárias, indo ao encontro das diretrizes da Reforma Psiquiátrica. É neste aspecto que Dimenstein, Lima e Macedo (2013) destacam que as ações de coordenação e continuidade de cuidados em saúde mental aqui propostas precisam ser pensadas como estratégia de inovação e fortalecimento da integralidade entre a Atenção Psicossocial e Atenção Básica em saúde. Para os autores a Atenção Primária e Psicossocial carecem do fortalecimento de tecnologias relacionais e que possam dar concretude à proposta de integralidade em saúde mental. Portanto, é de extrema relevância avançar com ações inovadoras na produção de cuidado em saúde mental, estabelecendo um novo modo de relacionar-se com as pessoas em situação de sofrimento psíquico e existencial voltado para a produção da vida.

Ao falar em integralidade em saúde mental significa sermos protagonistas de novas práticas, no sentido de agenciar e produzir novos territórios de encontros entre os profissionais, os serviços e a comunidade, que resultem no agir coletivo da produção do cuidado (Merhy, 2012) ou como bem qualificou Cecílio et al., (2006), no agir coletivo da formação de redes de cuidado. Por outro lado, percebe-se que apesar do engajamento dos atores que fazem parte do SUS, o princípio da integralidade foi o que menos avançou em termos de ganhar materialidade e expressividade nas práticas profissionais e no modo de organizar os serviços.

Contudo, os trabalhadores dos CAPS precisam assumir que o apoio matricial está entre um, dos vários dispositivos destacados pelo Ministério da Saúde (2003), através do apoio matricial busca-se superar a lógica da especialização e da fragmentação do trabalho no campo da saúde mental, por meio da responsabilização em conjunto dos casos, além de permitir regular o fluxo de pacientes nos serviços.

É importante destacar que o Apoio Matricial em saúde mental não pode ser reduzido como sendo, apenas, atenção aos usuários e capacitação/supervisão dos profissionais, pois se trata de uma tarefa complexa que, como foi colocado no decorrer deste trabalho, faz-se necessário a implementação de medidas que consolidem o AM, bem como uma política mais clara de assistência em saúde mental. Sendo, portanto, um equívoco considerar o AM como supervisão de caso, indo de encontro com a proposta principal do AM que é o envolvimento, a corresponsabilização pelos casos e troca de saberes entre os profissionais.

\section{Considerações Finais}

Vale destacar que os achados neste artigo não se encerram aqui, como proposta futura, maiores aprofundamentos podem ser realizados. No que se refere à saúde mental, identifica-se que ainda é necessário investir em estratégias que incluam essas ações no cotidiano da atenção básica. Pois o apoio matricial em saúde mental é considerado uma prática fundamental, 
uma vez que auxilia na condução de casos e situações complexas, bem como apoia o planejamento de ações e a educação permanente das equipes, dada à necessidade de estabelecer articulações necessárias para uma atenção resolutiva em saúde mental, superando os especialismos, pois permite fazer saúde de uma forma ampliada e integral.

Por fim, verificou-se que as implicações deste estudo para a área da saúde mental, são bastante relevantes. Pois, além de ampliar o olhar dos profissionais da saúde mental, através do conhecimento das equipes nas unidades básicas de saúde, sobre os usuários, famílias e território; também, propõe que os casos sejam de responsabilidade compartilhada. Neste sentido, o apoio matricial pode ser um regulador de fluxo, permitindo entender e diferenciar os casos que realmente precisam ser atendidos pela saúde mental, e os casos que podem ser acompanhados pela estratégia de saúde da família, ou pelo menos ser acolhidos momentaneamente por estes profissionais.

\section{Referências}

Bezerra, E. N. R. \& Dimenstein, M. D (2008). O Caps e o trabalho em rede: tecendo o apoio matricial na atenção básica. Psicol. cienc. prof. 28(3), 632-645 https://doi.org/10.1590/S1414-98932008000300015

Brasil. (2005). Ministério da Saúde. Secretaria de Atenção à Saúde. DAPE. Coordenação Geral de saúde mental. Reforma Psiquiátrica e política de saúde mental no Brasil. Documento apresentado à Conferência Regional de Reforma dos serviços de saúde mental: 15 anos depois de Caracas. Brasília: OPAS.

Brasil. (2007). Ministério da Saúde. Coordenação Geral de Saúde Mental. Saúde Mental no SUS: acesso ao tratamento e mudança do modelo de atenção. Relatório da gestão 2003- 2006. Brasília/DF.

Brasil. Ministério da Saúde. (2004). Secretaria de Atenção à Saúde. Coordenação Geral de Saúde Mental. Saúde Mental no SUS: os centros de atenção psicossocial. Brasília/DF, 2004.

Campos, G. W. S. \& Domitti, A. C. (2007). Apoio matricial e equipe de referência: uma metodologia para gestão do trabalho interdisciplinar em saúde, Cadernos de Saúde Pública, 23(2), 399-407. https://doi.org/10.1590/S0102-311X2007000200016

Campos, G. W. S. (2007). Subjetividade e administração de pessoal: considerações sobre modos de gerenciar o trabalho em equipes de saúde. In E.E Merhy \& R Onocko Campos (Eds.), Agir em saúde: um desafio para o público. Hucitec.

Cecílio, L. C. O., \& Matsmuto, N. F. (2006). Uma taxonomia operacional de saúde. In P. Roseni \& R. Mattos (Orgs.), Gestão em redes: tecendo os fios da integralidade em saúde. CEPESC.

Dias, J. V. S. \& Amarante, P. D. (2022). Educação Popular e Saúde Mental: Aproximando Saberes e Ampliando o Cuidado. Saúde em Debate, 46(132), 188199. https://saudeemdebate.org.br/sed/issue/view/51

Dias, J. V. S. \& Amarante, P. D. (2020). Saúde Mental e Educação Popular: possíveis diálogos. In C.A Paro, M.A.A, Lemões \& R, Pekelman (orgs.), Educação Popular e a (re)construção de práticas cuidadoras, 2, Editora do CCTA, 135-154. Coletânea Educação Popular em Saúde.

Dimenstein, M. D., Lima, A. I. \& Macedo, J. P. (2013). Integralidade em saúde mental: coordenação e continuidade de cuidados na atenção primária. In S Paulon \& R Neves (eds.), Saúde mental na atenção básica: a territorialização do cuidado. Sulina.

Dimenstein, M. D., Severo, A. K. S., Brito, M., Pimenta, A. L., Medeiros, V. \& Bezerra, E. N. R. (2009). O apoio matricial em unidades de saúde da família: experimentando inovações em saúde mental. Saude soc, 18(1), 63-74. https://doi.org/10.1590/S0104-12902009000100007

Feuerwerker, L. (2005). Modelos tecnoassistenciais, gestão e organização do trabalho em saúde: nada é indiferente no processo de luta para a consolidação do SUS. Interface Comunicação, Saúde e Educação, 9(18), 489-506.

Figueiredo, M. D. \& Onocko Campos, R. (2008). Saúde mental e atenção básica à saúde: o apoio matricial na construção de uma rede multicêntrica. Saúde Debate, 32(78-79-80), 143-149. https://www.redalyc.org/articulo.oa?id=406341773014

Leite, A. L. S. \& Paulon, S. (2013). Atenção básica e desinstitucionalização da loucura: acionando competências dos agentes comunitários de saúde. In S. Paulon \& R. Neves (Orgs.), Saúde mental na atenção básica: a territorialização do cuidado. Sulina.

Lima, A. F. (2018). (Re) pensando a saúde mental e os processos de desinstitucionalização. Histórias, intervenções e desafios ético-políticos. 15-224, Curitiba: Appris.

Martín-Baró, I. (1984). Guerra y Salud Mental. Estudios Centroamericanos, 429-430, 503-514.

Merhy, E. E. (2006) A rede básica como uma construção da saúde pública e seus dilemas. In E. E. Merhy \& R. Onocko (Orgs.), Agir em Saúde. Um desafio para o público. Hucitec.

Merhy, E. E. (2012). Saúde e direitos: tensões de um SUS em disputa, molecularidades. Saude soc, 21(2), 267-279. https://doi.org/10.1590/S010412902012000200002

Minayo, M. C. S. (2010). O desafio do conhecimento: pesquisa qualitativa em saúde. (12a ed. Hucitec. 
Research, Society and Development, v. 11, n. 3, e47511326634, 2022

(CC BY 4.0) | ISSN 2525-3409 | DOI: http://dx.doi.org/10.33448/rsd-v11i1.26634

Nascimento, C. C. (2007). Apoio matricial em saúde mental: possibilidades e limites no contexto da reforma psiquiátrica. Dissertação (Mestrado em Enfermagem). Universidade de São Paulo, São Paulo.

Natal. Secretaria Municipal de Saúde. (2005). Relatório Anual de Gestão.

OMS. (2005). Organização Mundial da Saúde. Cuidados inovadores para condições crônicas: componentes estruturais de ação - relatório mundial.

Pitta, A. (2001). Reabilitação Psicossocial no Brasil. São Paulo: Hucitec.

Rotelli, F. (2015). Formação e construção de novas instituições em saúde mental. In F. Basaglia., F. Rotelli \& G. Butti (Orgs.), Saúde mental, formação e crítica. Rio de Janeiro: Laps. p.37-50.

Yasui, S. (2010). Rupturas e encontros: desafios da reforma psiquiátrica brasileira. Editora Fiocruz. 